\title{
Using Light Scattering to Evaluate the Separation of Polydisperse Nanoparticles
}

\author{
Anne A. Galyean $^{\alpha}$, , Wyatt N. Vreeland ${ }^{\beta}$, James J. Filliben $^{\gamma}$, R. David Holbrook ${ }^{\delta}$, Dean C. Ripple ${ }^{\beta}$, \\ Howard S. Weinberg ${ }^{\alpha}$ \\ ${ }^{\alpha}$ Gillings School of Global Public Health, Department of Environmental Sciences and \\ Engineering, University of North Carolina, Chapel Hill, NC 27599, U.S.A. \\ ${ }^{\beta}$ Biomolecular Measurement Division, ${ }^{\gamma}$ Statistical Engineering Division, ${ }^{\delta}$ Materials Measurement \\ Science Division, National Institute of Standards and Technology, 100 Bureau Drive, \\ Gaithersburg, Maryland 20899, U.S.A. \\ *Corresponding author: galyean@live.unc.edu, phone: 1-208-521-9608
}

\section{Contact email addresses by author:}

Anne A Galyean - galyean@live.unc.edu

Wyatt N. Vreeland - wyatt.vreeland@nist.gov

James J. Filliben - james.filliben@nist.gov

R. David Holbrook - dave.holbrook@nist.gov

Dean C. Ripple - dean.ripple@nist.gov

Howard S. Weinberg - howard_weinberg@unc.edu

KEYWORDS: separation, multi angle light scattering, quasi-elastic light scattering, polydisperse, nanoparticle, flow field flow fractionation 


\begin{abstract}
The analysis of natural and otherwise complex samples is challenging and yields uncertainty about the accuracy and precision of measurements. Here we present a practical tool to assess relative accuracy among separation protocols for techniques using light scattering detection. Due to the highly non-linear relationship between particle size and the intensity of scattered light, a few large particles may obfuscate greater numbers of small particles. Therefore, insufficiently separated mixtures may result in an overestimate of the average measured particle size. Complete separation of complex samples is needed to mitigate this challenge. A separation protocol can be considered improved if the average measured size is smaller than a previous separation protocol. Further, the protocol resulting in the smallest average measured particle size yields the best separation among those explored. If the differential in average measured size between protocols is less than the measurement uncertainty, then the selected protocols are of equivalent precision. As a demonstration, this assessment metric is applied to optimization of cross flow $\left(V_{x}\right)$ protocols in asymmetric flow field flow fractionation (AF4) separation interfaced with online quasi-elastic light scattering (QELS) detection using mixtures of polystyrene beads spanning a large size range. Using this assessment metric, the $V_{x}$ parameter was modulated to improve separation until the average measured size of the mixture was in statistical agreement with the calculated average size of particles in the mixture. While we demonstrate this metric by improving AF4 $V_{x}$ protocols, it can be applied to any given separation parameters for separation techniques that employ dynamic light scattering detectors.
\end{abstract}

\title{
1. INTRODUCTION
}


Field flow fractionation (FFF) and specifically, flow field flow fractionation (FIFFF), has become one of the most favored methods for separating complex colloidal samples. FIFFF is a type of chromatography that does not require the use of a stationary phase and relies on hydrodynamic principles to separate particles in an open fluidic channel [1-4]. FlFFF can be directly interfaced with a wide variety of standard chromatography detectors, such as multi-angle light scattering (MALS) for particle sizing, quasi-elastic light scattering (QELS) for measuring particle diffusion coefficients, concentration detectors, fluorescence, or inductively coupled plasma-mass spectrometry for elemental analysis, among others [5]. FlFFF has been applied to particle separation of very small particles, such as humic substances in the $1 \mathrm{~nm}$ range, natural colloids in the $20-450 \mathrm{~nm}$ range, and larger particles, such as clay, in the 5-100 $\mu \mathrm{m}$ range [3]. FlFFF has been widely applied to nanoparticle analysis, such as metals, metal oxides, $\mathrm{SiO}_{2}$, and carbon black. Additionally, it has been used to analyze complex sample matrices such as soil suspensions and colloids in fresh and marine water samples [6] while simultaneously reducing sample complexity and fractionating colloidal materials by size. There is a growing body of literature on how to optimize and define AF4 separation parameters for various applications and types of nanomaterials, based on theory and experimental parameters that influence AF4 $[3,7-$ 13].

The development of separation techniques operated in tandem with MALS and QELS detectors allows for size and molar mass distribution measurements of arbitrary polydisperse mixtures of particles and in some cases, information about molecular conformation [14]. While the angular dependence of scattered light can provide information about particle size as measured by MALS, QELS directly measures the translational diffusion coefficient, and computes hydrodynamic radius $\left(R_{h}\right)$ using the Stokes-Einstein equation. When light scattering is combined with a nondestructive separation technique that presents the light scattering detector with scattering from an 
essentially monodisperse particle size at each measured fraction, the size distribution of the original sample can be calculated $[15,16]$. Because the relationship between particle size and scattering intensity is highly non-linear ( $\mathrm{I} \propto \mathrm{r}^{6}$, where $I$ is the scattering intensity and $r$ is the particle radius; light scattering theory has been described in detail elsewhere [15-17]), the measured size can be heavily biased to being erroneously large in insufficiently separated mixtures. In other words, a few large particles may obscure the detection and accurate measurement of much greater numbers of small particles. Therefore, obtaining an accurate measurement of a polydisperse distribution requires a separation of sufficient resolution, and the optimum separation will result in the smallest average size (Figure 1).

Separations of various natural, environmental, biological, or otherwise complex samples are less defined and contain a higher level of variability $[14,18-20]$ when compared to the separations of mixtures of monodisperse or distinct particle sizes. Currently, to our knowledge, there is no metric to establish whether the separation optimization is complete and the separation is the best possible given the available experimental conditions. Experience and FFF theory can provide tools for choosing separation conditions based on a certain range of particle sizes. However, in the case of unknown or complex mixtures spanning a large size range, these conditions may be much less obvious. Similarly, extremely polydisperse mixtures, such as environmental or natural samples, may not yield sufficient resolution between sample components to judge separation quality by observing the raw data in fractograms alone. Therefore, the analysis of a single separation and its data may not provide adequate information about the accuracy of the measurement. Thus, a rigorous characterization requires multiple separation protocols and comparison among them. 
To address the challenges described above, we compare the average measured particle size for a distribution of particles with various separation parameters, and demonstrate that the best separation possible is obtained when the average size is at a minimum. In this work, the separation parameter of cross flow in an AF4-QELS system is used as a simplified example to represent changing separation conditions. This separation metric is described and subsequently applied to mixtures of polystyrene nanoparticles of several known mean sizes to mimic complex mixtures.

\section{MATERIALS AND METHODS ${ }^{1}$}

\subsection{INSTRUMENTATION}

AF4 was performed using an Eclipse DualTec separation module (Wyatt Technologies Corp., Santa Barbara CA) with OpenLab CDS Chem Station edition software (Agilent Technologies, Santa Clara, CA). Injections were made with an Agilent 1260 Infinity series isopump and autosampler with a $900 \mu \mathrm{L}$ injection loop. The run buffer was degassed by a Gastorr TG-14 (Flom USA, San Diego, CA, USA) at $10 \mathrm{kPa}$ directly from solvent bottles, and filtered in series by a polytetrafluoroethylene frit (RESTEK Corp., Bellefontane, PA, USA) and a $0.1 \mu \mathrm{m}$ Durapore ${ }^{\circledR}$ membrane filter (Millipore Inc., Billerica, MA, USA). Separation was performed with

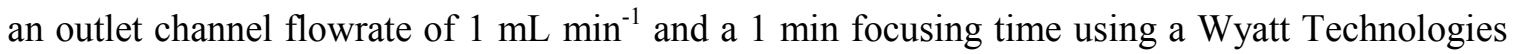
"short channel" containing a $350 \mu \mathrm{m}$ spacer and a regenerated cellulose ultrafiltration membrane with $5 \mathrm{kDa}$ cutoff (Wyatt Technologies Corp.). AF4 instrumental parameters held constant for each protocol are provided in Table 1.

\footnotetext{
${ }^{1}$ Certain commercial equipment, instruments, or materials are identified in this paper in order to specify the experimental procedure adequately. Such identification is not intended to imply recommendation or endorsement by the National Institute of Standards and Technology, nor is it intended to imply that the materials or equipment identified are necessarily the best available for the purpose.
} 
Quasi electric light scattering (QELS) measurements were made with a WyattQELS ${ }^{\mathrm{TM}}$ detector through a QELS fiber attached to the Wyatt DAWN ${ }^{\circledR}$ II MALS detector at a nominal angle of $140^{\circ}$ with a $2.0 \mathrm{sec}$ collection interval (Wyatt Technologies Corp.). Multi angle light scattering (MALS) measurements were not included in this work. Polystyrene samples were prepared in the ammonium nitrate buffer and placed in amber glass vials sealed with PTFE/silicone septa (Chemglass Life Sciences, Vineland, NJ, USA). Data were collected and analyzed with ASTRA $^{\text {TM }}$ Software version 6.1.1.17. ASTRA ${ }^{\mathrm{TM}}$ Software calculates the $\mathrm{R}_{\mathrm{h}}$ using QELS data by measuring the time-dependent fluctuations in scattered light. The fluctuations are then directly related to the rate of diffusion of the molecule through the solvent, which, in turn, is related to the particles' hydrodynamic radii. In this work, the "average $\mathrm{R}_{\mathrm{h}}$ " refers to the uncertainty-weighted average, determined in the ASTRA software by:

$$
\left(R_{h}\right)_{a v g}=\frac{\sum r_{i} \sigma_{r_{i}}^{2}}{\sum \sigma_{r_{i}}^{2}}
$$

where $r_{i}$ represents the calculated $\mathrm{R}_{\mathrm{h}}$ of the $i$ th time slice, and $\sigma_{r_{i}}$ is the uncertainty in the radius measurement, defined as:

$$
\sigma_{r_{\text {avg }}}=\frac{1}{\sum \sigma_{r_{i}}^{2}}
$$

\subsection{MATERIALS}

The AF4 run buffer was prepared by dissolving ammonium nitrate (Sigma-Aldrich, St. Louis, MO, USA) in laboratory-grade $18.2 \mathrm{M} \Omega \cdot \mathrm{cm}$ water with $0.01 \%$ sodium azide (Ricca Chemical Company, Arlington, TX, USA) as an antimicrobial. Mixtures of spherical Nanosphere ${ }^{\mathrm{TM}}$ NIST- 
traceable polystyrene beads (Thermo Scientific, Waltham, MA, USA) of various sizes in laboratory-grade $18.2 \mathrm{M} \Omega \cdot \mathrm{cm}$ water were prepared. Two mixtures were prepared from bead suspensions of known bead size and concentration. Sample 1, prepared to represent a mixture containing a large amount of small particles and a small amount of large particles, consisted of a mixture of $(21 \pm 2) \mathrm{nm}\left(\left[8.25 \mathrm{mg} \mathrm{mL}^{-1}\right]\right),(41 \pm 4) \mathrm{nm}\left(\left[0.47 \mathrm{mg} \mathrm{mL}^{-1}\right]\right),(57 \pm 4) \mathrm{nm}([0.11 \mathrm{mg}$ $\left.\left.\mathrm{mL}^{-1}\right]\right),(81 \pm 3) \mathrm{nm}\left(\left[0.05 \mathrm{mg} \mathrm{mL}^{-1}\right]\right)$ and $(100 \pm 3) \mathrm{nm}\left(\left[0.03 \mathrm{mg} \mathrm{mL}^{-1}\right]\right)$ certified diameter beads with manufacturer specified size distribution. Sample 2, prepared to simulate polydisperse samples containing low scattering signal from small particles and large scattering signal from large particles, consisted of a mixture containing spheres of $(21 \pm 2) \mathrm{nm},(41 \pm 4) \mathrm{nm},(57 \pm 4)$ $\mathrm{nm},(81 \pm 3) \mathrm{nm}$ certified mean diameters, each at a particle concentration of $2.7 \times 10^{12} \mathrm{~mL}^{-1}$. Polystyrene bead mean diameters were provided by the supplier and confirmed in-house by both dynamic light scattering and AF4-QELS (data not shown).

\subsection{METHODS}

Polystyrene sphere mixtures were separated with a range of low, medium, and high particle retention AF4 separation protocols. Cross flow $\left(V_{x}\right)$ for all protocols was ramped down linearly from the starting $V_{x}$ rate to $0 \mathrm{~mL} / \mathrm{min}$ over the period of elution. At a channel flow of $1 \mathrm{~mL} / \mathrm{min}$, the maximum $V_{x}$ allowed with the instrument setup is $2.99 \mathrm{~mL} \mathrm{~min}^{-1}$. From low to high retention, protocols were defined with initial $V_{x}$ rates of $0.50 \mathrm{~mL} \mathrm{~min}^{-1}, 1.00 \mathrm{~mL} \mathrm{~min}{ }^{-1}, 1.25 \mathrm{~mL} \mathrm{~min}^{-1}, 1.50$ $\mathrm{mL} \min ^{-1}, 1.75 \mathrm{~mL} \min ^{-1}, 2.00 \mathrm{~mL} \min ^{-1}, 2.25 \mathrm{~mL} \min ^{-1}, 2.50 \mathrm{~mL} \mathrm{~min}{ }^{-1}, 2.75 \mathrm{~mL} \mathrm{~min}$, and 2.99 $\mathrm{mL} \min ^{-1}$, and linearly decreasing the $V_{x}$ to $0 \mathrm{~mL} \min ^{-1}$ over the gradient duration. Duration of the $V_{x}$ gradient from low to high retention were $10 \mathrm{~min}, 20 \mathrm{~min}, 30 \mathrm{~min}, 40 \mathrm{~min}, 50 \mathrm{~min}$, and $60 \mathrm{~min}$. Figure 2 is a graphical representation of the initial $V_{x}$ rate and ramp times for each protocol used in this work. Particle size analysis was performed at each data point across the entire elution 
window, from the point of injection through end of the separation. To assess the quality of a given separation relative to another, the average $R_{h}$ of the measured sample distribution was calculated for each parameter setting using all data within the AF4 elution window. Using the uncertainty-weighted average, points of lower accuracy with low signal to noise ratios are less influential in calculating $\mathrm{R}_{\mathrm{h}}$, and no statistical manipulation was required. Further, all data were used to maintain consistency between protocols of different elution lengths and resolution. Each protocol was run in triplicate to ensure the reproducibility of the separation.

\section{RESULTS AND DISCUSSION}

\subsection{AF4 SEPARATION OF POLYSTYRENE MIXTURES}

The separation metric was applied to polystyrene bead mixtures spanning a wide dynamic range (20-100 nm) in increments of $20 \mathrm{~nm}$. While polystyrene beads are inherently less polydisperse than a complex natural sample, these mixtures mimic complex samples in that baseline resolution was not possible by adjusting the $\mathrm{V}_{\mathrm{x}}$ and $\mathrm{V}_{\mathrm{x}}$ gradient alone within the designated elution window. Polystyrene beads (particles) are often used as model systems for AF4 size calibration and system optimization prior to analysis with real, complex samples $[2,3,21,22]$ and polystyrene standards separated with AF4 have been shown to have very good sample recoveries [21]. The application of this metric to more complex samples, like environmental samples, would require additional characterization and recovery analysis to account for non-ideal behavior, such as the potential risk of particle-membrane and particle-particle interactions, to identify any sample changes that may occur with separation parameter modification. Recovery experiments are typically performed with the addition of a concentration detector, like refractive index or UV-Vis spectrophotometry. 
Samples 1 and 2 were separated by AF4-QELS using protocols of increasing retention time, shown in Figure 2 and described below. For demonstration purposes, the separation parameters chosen were $V_{x}$ and $V_{x}$ gradient time, as they are primary AF4 separation parameters. While we have chosen two separation parameters out of a potentially larger set of instrument factors as a demonstration, the proposed methodology could be applied to any combination of other parameters or potentially all could be used simultaneously, depending on the desired application. In the case of all available parameters being applied, a broader optimized methodology of screening and optimization designs along with appropriate analysis would be required.

The analysis procedure chosen for this work is as follows: beginning with the protocol of very low retention time (protocol: " $A$ "), the average measured size for each sample separated using each protocol of increasing retention time (protocols: " $B$ " through " $K$ ") was compared with that of the previous protocol until increasing retention no longer produced a significant decrease in average measured size. At this point, a protocol of higher retention in an orthogonal direction of steepest descent to that of previous pair was selected (protocols: " $P_{o l}$ and $P_{o 2}$ "). Exploring $P_{o l}$ and $P_{o 2}$ better populates the experimental space in identifying a local minimum in average measured size. All values for each protocol were plotted for both mixtures, as shown in Figure 3a for Sample 1 and Figure $3 \mathrm{~b}$ for Sample 2. In the event that increasing particle retention by turning up the $V_{x}$ and elution ramp did not yield a significant decrease in average $R_{h}$ compared to the previous protocol, the value was compared to that of $P_{\max }$ to ensure that an extreme increase in retention would not result in a significantly lower average $R_{h}$. If this comparison failed, then the retention was increased and the process repeated until the statistical minimum was identified. A thorough, statistical methodology for this approach is detailed in the Supporting Materials (Figure S-3). 


\subsection{LIGHT SCATTERING ANALYSIS}

In both cases, protocol $H$ was identified as being the optimal protocol of those tested, with the average measured $R_{h}$ found to be $(21.1 \pm 0.5) \mathrm{nm}$ and $(26.3 \pm 0.6) \mathrm{nm}$ for the Samples 1 and 2 , respectively (Figure 3). All uncertainties are defined at the $95 \%$ confidence interval. The average measured $R_{h}$ and associated uncertainty for Samples 1 and 2 separated with protocols $A-K, P_{o l}$, $P_{o 2}$, and $P_{\max }$ for $n=3$ are given in the Supporting Materials (Table S-1) and shown relative to protocol selection in Figure 4.

The calculated average $R_{h}$ of the polystyrene mixtures was $(21.7 \mathrm{~nm} \pm 1.0 \mathrm{~nm})$ for Sample 1, and $(23.6 \mathrm{~nm} \pm 1.5) \mathrm{nm}$ for Sample 2, based on the known particle size distribution and individual mean particle sizes for each polystyrene bead in the mixture as determined by light scattering. The calculations are provided in the Supporting Materials (Table S-2). While separation expertise might suggest that the optimal parameters lies after the midpoint of increasing retention strength and could have been used as a more efficient initial starting point for either design or analysis, we chose to begin at low retention in order to better demonstrate the trend towards a minimum average measured size.

It is expected that the average measured size for Sample 1 is lower than that of the Sample 2, as the majority of QELS signal intensity in Sample 2 is of very low intensity compared to signal from large particles. The signal from the low amount of scatter attributed to the smaller particle sizes in Sample 2 might be too low to be distinguished from the larger particles and, therefore, the measured average size will be larger than the actual average size. 


\subsection{SEPARATIONS OF POLYDISPERSE MIXTURES}

In the case of polydisperse samples with characteristics similar to Sample 2, it may not be possible to detect the smallest sizes within the constraints of the instrument; either due to detection limits from low scattering intensity of small particles, or further increases in retention time causes dispersive mechanisms to reduce the separation efficiency. In contrast, Sample 1 contains a relatively large amount of small particles compared to large particles (approximately $98 \%$ of polystyrene mass concentration contains particles between $\sim 20 \mathrm{~nm}$ and $\sim 40 \mathrm{~nm}$ in diameter, whereas the remaining $2 \%$ of polystyrene mass concentration contains particles between $\sim 60 \mathrm{~nm}$ and $\sim 100 \mathrm{~nm}$ in diameter), thus, there is more scattering signal from the smaller particles relative to larger particles. In a complex mixture, achieving baseline resolution between particle species is unlikely. By minimizing the measured size, however, the ability to find the separation protocol that yields the best possible measurement considering the polydisperse sample distribution and the instrument operational limits. These observations are demonstrated in the representative fractograms and hydrodynamic radius distribution overlays, given in Figure 5, for Samples 1 and 2 using separation protocols $A, H$, and $P_{\max }$.

The statistical details for a stepwise approach for selecting a successful separation protocol, and the determination as to whether the chosen protocol is sufficient for the intended application is shown via flowchart and a worked example in the Supporting Materials (Figure S-3 and Table S4, respectively). While this paper identifies a local optimum protocol based on discrete data, the more rigorous approach would be to identify a global optimum based on a continuous surface design of measured particle size against separation parameters along a continuum of settings. The cost of global optimization would require significantly more data. 
The point of optimal separation identified by statistically significant decreases in measured size may not in fact be the point of the very best separation. However, increasing retention time will not likely produce a statistically smaller average size than the protocol with shorter retention time, as demonstrated with Samples 1 and 2 beyond the local minimum of Protocol $H$, and will require an increase in analysis time with minimal improvement in separation. Furthermore, higher retention may also increase the average measured size as dispersive effects compromise the benefits of increased selectivity.

Optimization depends on the relative priority of retention time and separation selectivity. Depending on the sample properties and the intended application, a separation protocol can be selected that balances analysis time and separation resolution. The separation variables, $V_{x}$ and ramp time, were chosen to linearly populate the domain of particle retention from the protocol of lowest retention $(A)$ to one of highest retention $\left(P_{\max }\right)$, as shown in Figure 2. Simple separation theory predicts that the average measured size will decrease to an asymptote; beyond a certain separation, additional retention increases do not yield a significant decrease in measured size. In reality, however, the average measured size will typically reach a minimum and then (with higher retention) likely increase due to dispersive effects.

\subsection{METHOD APPLICATION}

There are several options for the sequential choice of protocols. For a stepwise approach without making assumptions based on sample distribution, a protocol should be initially chosen with minimum retention, while the subsequent protocols of increasing retention should be selected so that the average measured size decreases significantly before reaching a minimum. Again, in situations where separation theory and experience are not sufficient for qualitatively determining 
the separation quality of complex, polydisperse mixtures, more objective and rigorous approaches should be applied. In most cases, a gradient method can be applied to best identify a local minimum within the experimental space, by analyzing protocols orthogonal to those identified as optimal in the direction of steepest descent [24, 25], e.g., $P_{o 1}$ and $P_{o 2}$ for protocol $H$ in Figure 2. The larger design issue in regard to choosing the number of parameters to investigate balanced with practical constraints of how many sample runs are affordable is the subject of further study. The next step is to perform a formal response surface optimization analysis, a methodology to explore the relationships between several independent variables and one or more response variable [26]. A response surface design would provide a more statistically rigorous way of identifying globally optimal parameters that yield the minimum average measured size, especially for the cases where the optimal set of parameters might not lie directly on the linear path between a set of parameters with very low retention and $P_{\max }$.

\section{CONCLUSIONS}

This work introduces a new metric for optimizing separations of polydisperse samples through a demonstration using AF4-QELS with a model system of polystyrene bead mixtures in order to mimic complex samples. The comparison of average measured size between separation parameters provides an analytical means for objectively converging to the optimal combination of instrumental parameters and identifying a separation protocol that sufficiently separates a polydisperse mixture of nanoparticles, especially when separation theory and observations of the raw data become difficult to apply to complex samples. By identifying a separation protocol that is of sufficient selectivity, large and small particles are separated in such a way that they are detected distinctly, resulting in an overall lower average particle size measurement. Therefore, the more optimal the separation, the smaller the average measured size, providing a metric for 
optimizing separation of polydisperse samples of unknown size distributions. Additionally, this metric establishes a methodology of identifying a quality separation while minimizing unnecessary analysis time. Although demonstrated using AF4-QELS, the separation metric and step-wise approach are applicable to any chromatography technique coupled with dynamic light scattering detection.

\section{ACKNOWLEDGEMENTS}

This material is based upon work supported by the National Science Foundation Graduate Research Fellowship Program under Grant No. (DGE-1144081). Any opinions, findings, and conclusions or recommendations expressed in this material are those of the authors and do not necessarily reflect the views of the National Science Foundation. This research was supported, in part, by funds from the National Institutes of Health (T32ES007018) and through an Off-Campus Dissertation Research Fellowship from the University of North Carolina at Chapel Hill. The authors also acknowledge helpful discussions with John Pettibone, Ph.D., of the National Institute of Standards and Technology in the preparation of this manuscript.

\section{References}

1. Messaud, F.A.; Sanderson, R.D.; Runyon, J.R.; Otte, T.; Pasch, H.; Williams, S.K.R. An overview on field-flow fractionation techniques and their applications in the separation and characterization of polymers. Prog. Polym. Sci. 2009, 34, 351-368. 

characterization of nanoparticles in complex food and environmental samples by field-flow fractionation. TrAC-Trend. Anal. Chem. 2011, 30, 425-436.

3. Baalousha, M.; Stolpe, B.; Lead, J.R. Flow field-flow fractionation for the analysis and characterization of natural colloids and manufactured nanoparticles in environmental systems: A critical review. J. Chromatogr. A 2011, 1218, 4078-4103.

4. Lattuada, M.; Olivo, C.; Gauer, C.; Storti, G.; Morbidelli, M. Application of Asymmetric Flow-Field Flow Fractionation to the Characterization of Colloidal Dispersions Undergoing Aggregation. Langmuir 2010, 26, 7062-7071.

5. Bednar, A.J.; Poda, A.R.; Mitrano, D.M.; Kennedy, A.J.; Gray, E.P.; Ranville, J.F.; Hayes, C.A.; Crocker, F.H.; Steevens, J.A. Comparison of on-line detectors for field flow fractionation analysis of nanomaterials. Talanta 2013, 104, 140-148.

6. Sermsri, W.; Jarujamrus, P.; Shiowatana, J.; Siripinyanond, A. Flow field-flow fractionation: a versatile approach for size characterization of alpha-tocopherol-induced enlargement of gold nanoparticles. Anal. Bioanal. Chem. 2010, 396, 3079-3085.

7. Gigault, J.; Pettibone, J.M.; Schmitt, C.; Hackley, V.A. Rational strategy for characterization of nanoscale particles by asymmetric- flow field flow fractionation: A tutorial. Anal. Chim. Acta 2014, 809, 9-24.

8. Bolea, E.; Jimenez-Lamana, J.; Laborda, F.; Castillo, J.R. Size characterization and quantification of silver nanoparticles by asymmetric flow field-flow fractionation coupled with inductively coupled plasma mass spectrometry. Anal. Bioanal. Chem. 2011, 401, 2723-2732.

9. Hawe, A.; Romeijn, S.; Filipe, V.; Jiskoot, W. Asymmetrical flow field-flow fractionation method for the analysis of submicron protein aggregates. J. Pharm. Sci. 2012, 101, 4129-4139.

10. Thang, N.; Geckeis, H.; Kim, J.; Beck, H. Application of the flow field flow fractionation (FFFF) to the characterization of aquatic humic colloids: evaluation and optimization of the method. Colloid Surf. A-Physicochem. Eng. Asp. 2001, 181, 289-301.

11. Hupfeld, S.; Ausbacher, D.; Brandl, M. Asymmetric flow field-flow fractionation of liposomes: optimization of fractionation variables. J. Sep. Sci. 2009, 32, 1465-1470.

12. Loeschner, K.; Navratilova, J.; Legros, S.; Wagner, S.; Grombe, R.; Snell, J.; von der Kammer, F.; Larsen, E.H. Optimization and evaluation of asymmetric flow field-flow fractionation of silver nanoparticles. J. Chromatogr. A 2013, 1272, 116-125.

13. Lyven, B.; Hassellov, M.; Haraldsson, C.; Turner, D. Optimisation of on-channel preconcentration in flow field-flow fractionation for the determination of size distributions of low molecular weight colloidal material in natural waters. Anal. Chim. Acta 1997, 357, 187-196. 
14. von der Kammer, F.; Baborowski, M.; Friese, K. Field-flow fractionation coupled to multiangle laser light scattering detectors: Applicability and analytical benefits for the analysis of environmental colloids. Anal. Chim. Acta 2005, 552, 166-174.

15. Wyatt, P.J. Submicrometer Particle Sizing by Multiangle Light Scattering following Fractionation. J. Colloid Interface Sci. 1998, 197, 9-20.

16. Wyatt, P.J. Light scattering and the absolute characterization of macromolecules. Anal. Chim. Acta 1993, 272, 1-40.

17. Fabelinskiı̌, I.L. Molecular Scattering of Light. Plenum Press: New York, 1968.

18. Popovici, S.; Kok, W.; Schoenmakers, P. Band broadening in size-exclusion chromatography of polydisperse samples. J. Chromatogr. A 2004, 1060, 237-252.

19. Filella, M.; Zhang, J.; Newman, M.E.; Buffle, J. Analytical applications of photon correlation spectroscopy for size distribution measurements of natural colloidal suspensions: capabilities and limitations. Colloids Surf. Physicochem. Eng. Aspects 1997, 120, 27-46.

20. Giddings, J. Field-Flow Fractionation - Analysis of Macromolecular, Colloidal, and Particulate Materials. Science 1993, 260, 1456-1465.

21. Otte, T.; Bruell, R.; Macko, T.; Pasch, H.; Klein, T. Optimisation of ambient and high temperature asymmetric flow field-flow fractionation with dual/multi-angle light scattering and infrared/refractive index detection. J. Chromatogr. A 2010, 1217, 722-730.

22. Makan, A.C.; Otte, T.; Pasch, H. Analysis of High Molar Mass Branched Polybutadienes by SEC-MALLS and AF4-MALLS. Macromolecules 2012, 45, 5247-5259.

23. Pitkaenen, L. and Striegel, A.M. AF4/MALS/QELS/DRI characterization of regular star polymers and their "span analogs". Analyst 2014, 139, 5843-5851.

24. Bortz, D.M. and Kelley, C.T. The Simplex Gradient and Noisy Optimization Problems, In Computational Methods for Optimal Design and Control, 24th ed.; Borggaard, J., Burns, J., Cliff, E. and Schreck, S., Eds.; Birkhauser Boston: Cambridge, MA, 1998; Vol.24 pp. 77.

25. Snyman, J. Practical Mathematical Optimization: An Introduction to Basic Optimization Theory and Classical and New Gradient-Based Algorithms. Springer Science + Business Media, Inc: New York, NY, 2005.

26. Box, G.E.P.; Hunter, W.G.; Hunter, J.S. Statistics for Experimenters: An Introduction to Design, Data Analysis and Model Building. Wiley: New York, 1978. 
2

3

Figure 1. Cartoon depicting separation scenarios for a complex mixture of particles: (Left) insufficiently separated sample where large particles dominate the measurement and obscure smaller particles from detection, and (Right) a size-based separation of sufficient selectivity where both large and small particles are detected separately.

Figure 2. $\mathrm{V}_{\mathrm{x}}$ and elution time of selected protocols using AF4-QELS along the trend of increasing retention strength from $A-K . P_{o 1}$ and $P_{o 2}$ represent protocols that are orthogonal to protocol $\mathrm{H}$ in separation time and initial $V_{x} . P_{\max }$ represents the protocol of highest retention possible given instrumental or experimental limitations.

Figure 3. The combined average measured $R_{h}(\diamond)$ and associated uncertainty from (a) Sample 1 (b) Sample 2 with protocols $A-K, P_{o 1}, P_{o 2}$, and $P_{\max }$ for $n=3$ using AF4-QELS. The optimal protocol is " $H$ " (ם), where $R_{h}$ is minimized. The calculated average $R_{h}$ and associated uncertainty 
based on the actual size distribution for each sample is given as the dashed line. All uncertainties are defined at the $95 \%$ confidence interval. $R_{h}=$ Hydrodynamic Radius (nm).

Figure 4. The average measured $R_{h}$ and associated uncertainty for (a) Sample 1 and (b) Sample 2 separated with selected protocols, shown as initial cross flow vs. ramp time, for $n=3$. $R_{h}=$ Hydrodynamic Radius (nm).

Figure 5. Representative fractograms from Sample 1 using separation protocol (a) $A$, (b) $H$, and (c) $P_{\max }$ Fractograms from Sample 2 are also shown (d). Sample 2, Protocol A resulted in detector saturation. The solid line represents the relative light scattering signal intensity and the markers indicate the measured hydrodynamic radius (nm) at each time slice. 
AF4 Operating Conditions

\begin{tabular}{ll}
\hline Membrane & Regenerated cellulose, $10 \mathrm{kDa} \mathrm{MWCO}$ \\
Carrier liquid & $1.0 \mathrm{mM} \mathrm{NH}_{4} \mathrm{NO}_{3}$ in $18.2 \mathrm{M} \Omega$ nanopure water \\
Spacer thickness & $350 \mu \mathrm{m}$ \\
Detector flow & $1.00 \mathrm{~mL} / \mathrm{min}$ \\
& $50 \mu \mathrm{L}$ (Sample 1) \\
Injection volume & $60 \mu \mathrm{L}$ (Sample 2) \\
Injection flow & $0.20 \mathrm{~mL} /$ min \\
& $1.00 \mathrm{~min}$ focus \\
Focusing Regime & $3.00 \mathrm{~min}$ focus + inject \\
& $2.00 \mathrm{~min}$ focus \\
Focusing Flow & $1.50 \mathrm{~mL} / \mathrm{min}$ \\
\hline \hline
\end{tabular}




$$
\text { (c) }
$$


Fure 2

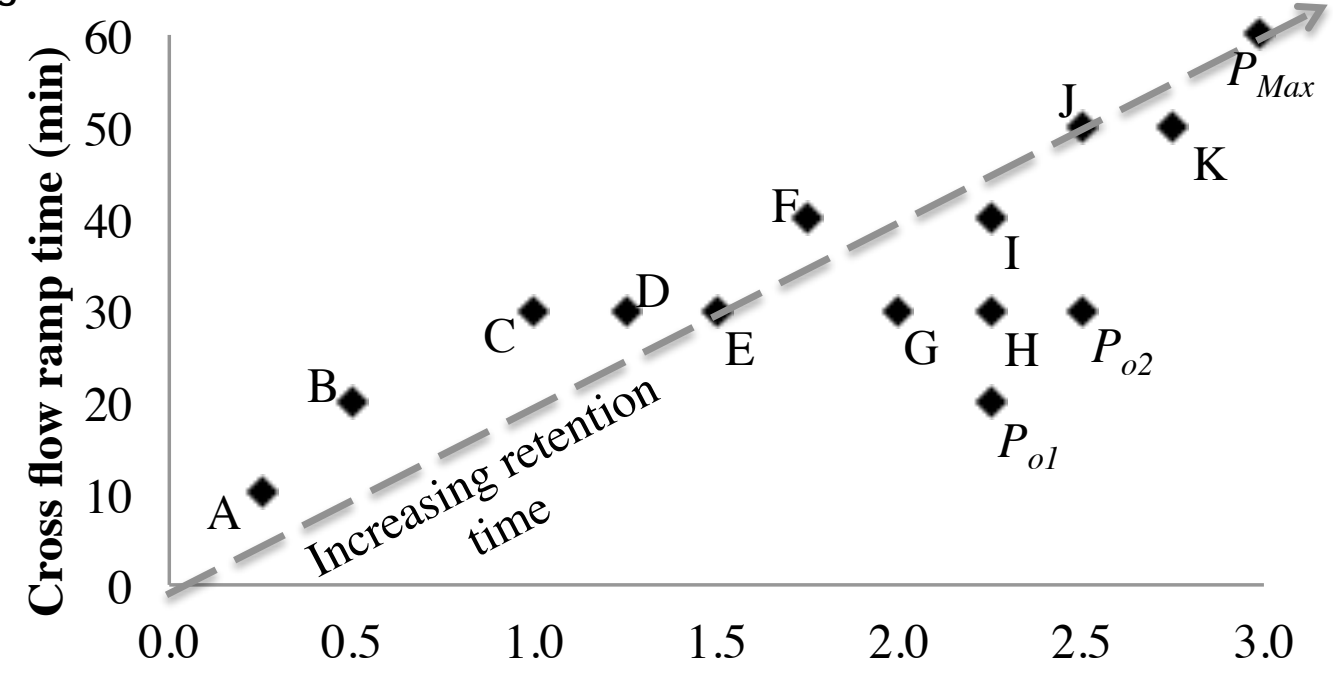

Initial cross flow rate (mL min-1) 


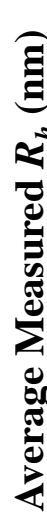

34
32
30
28
26
24
22
20
18

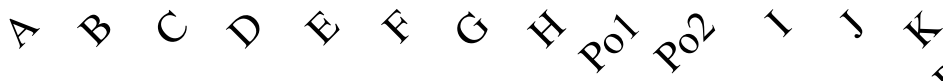

\section{Protocol}

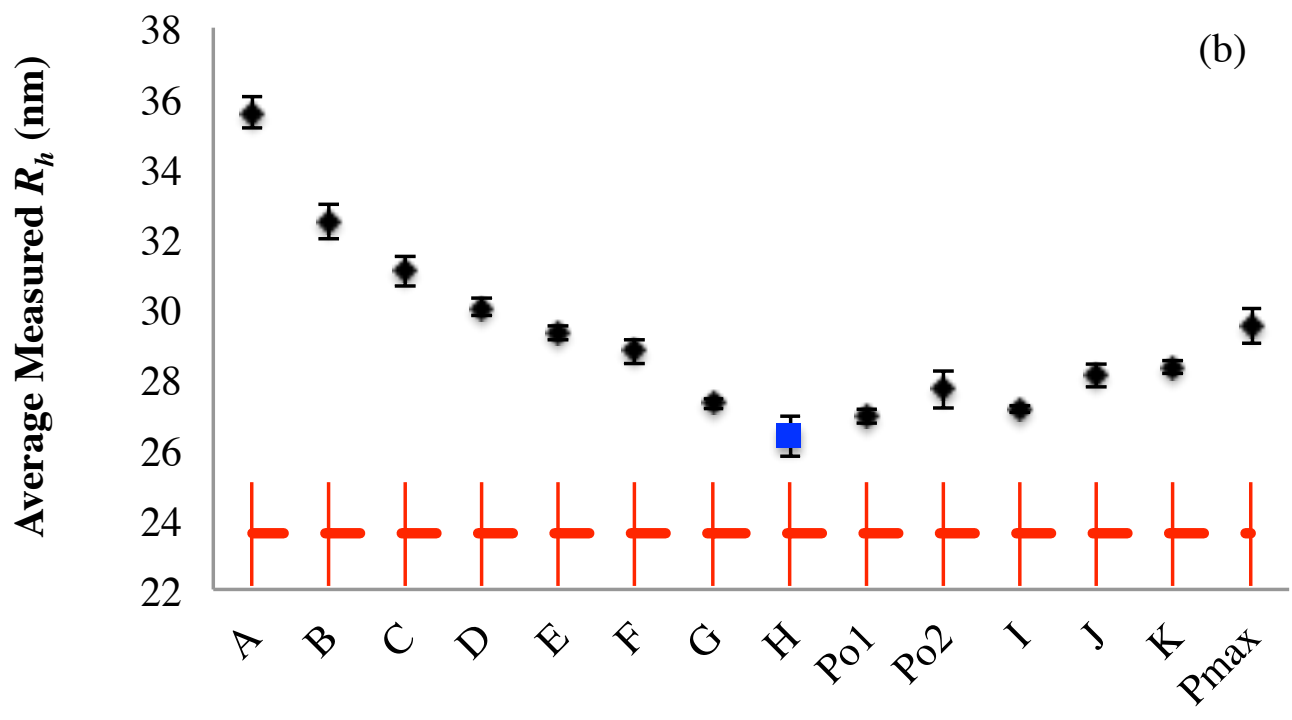


Figure $4 a$

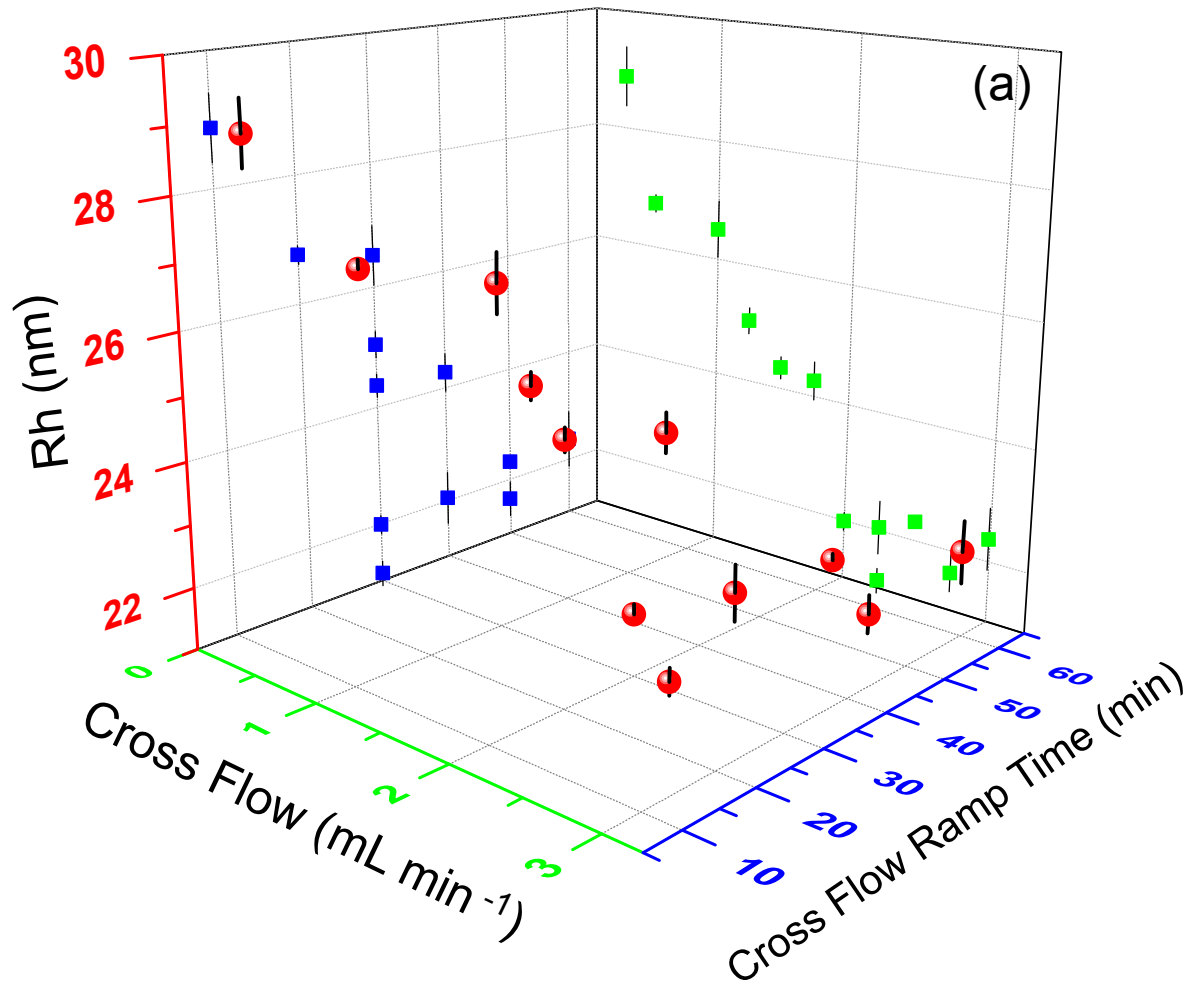


Figure 4b

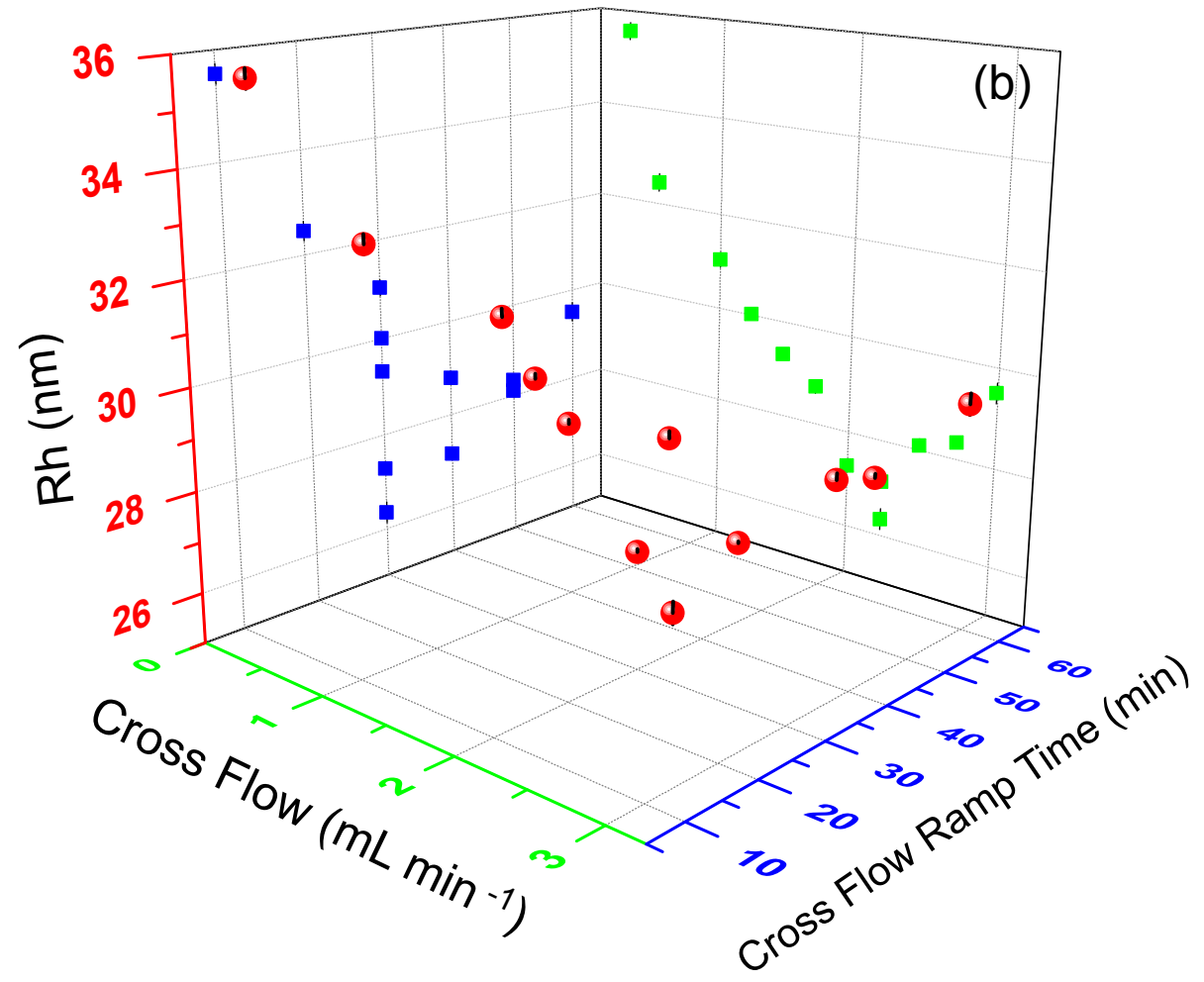




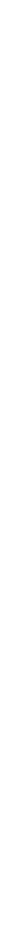


Figure 5b

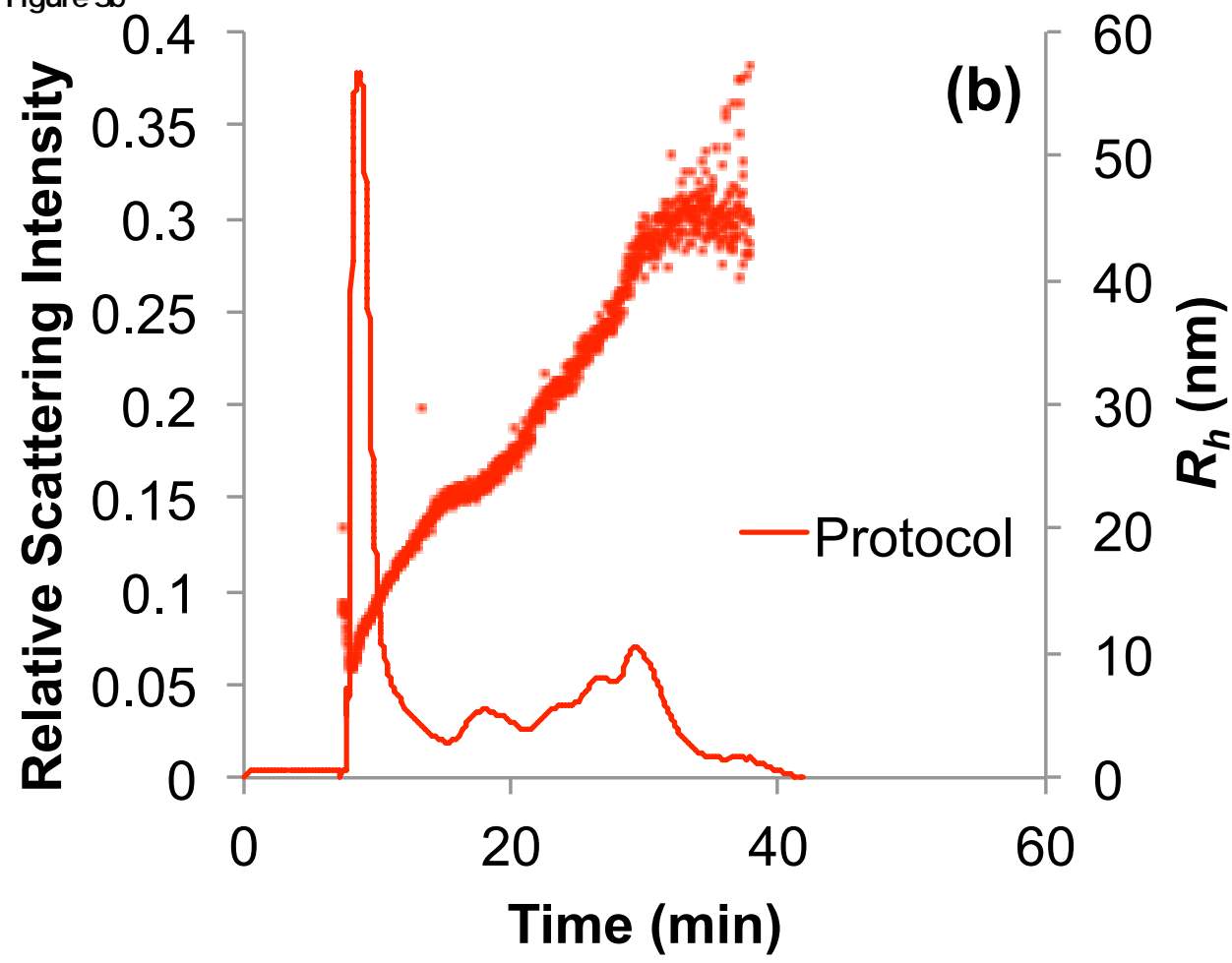




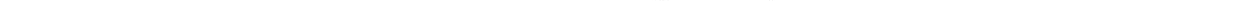


